\title{
Empirical Study on Performance Evaluation Between Long Term Evolution (LTE), Third Generation (3G) and TV White Space Availability for Wireless Campus Network
}

\author{
Rosdiadee Nordin, Anabi Hilary Kelechi, Mohd Hazree Easa, Syahiran Ahmad, Sameh Musleh \\ Department of Electrical, Electronic and Systems Engineering \\ Faculty of Engineering and Built Environment \\ Universiti Kebangsaan Malaysia \\ 43600, Bangi, Selangor, MALAYSIA \\ e-mail: adee@ukm.edu.my, kelana05@yahoo.com,syeranahmad@gmail.com,sameh.musleh@gmail.com
}

\begin{abstract}
University campuses are increasingly relying on wireless communication as the preferred media to access the web. Hence, there is a need to conduct on site performance evaluation on the existing wireless standards with the view of analyzing their expected and actual performance. In this paper, a drive-test was performed on three (3) wireless standards of LTE Release 8, 3G and Television White Space (TVWS). The drive-test was performed in both in-building and outdoor environments in a sub-urban campus settings. Results from LTE Release 8, 3G indicate that user mobility affects the received user throughput and Reference Signal Strength Indicator (RSSI). The test cases are executed by using a real LTE user equipment, equipped with ASCOM's TEMS software on a live 3G and 4G mobile networks for the both Malaysian mobile service providers; Maxis and Celcom. The results showed sufficient coverage within the campus area from both operators. On the other hand, results from field experiment indicate that TVWS is available on the campus. TVWS presents significant new opportunities within the context of emerging 4G networks for deploying new wireless access technology capable of meeting students expectation in terms of throughput and increasing broadband access for the fraction when the legacy $4 \mathrm{G}$ networks experience coverage blackout.
\end{abstract}

Keywords: coverage; drive test; HSPA+; LTE Release 8; mobile network; performance metrics; TEMS, TV White Space

\section{INTRODUCTION}

Performance study of wireless mobile networks has been on the increase and is driven by spectrum utilization and expected-actual data rate. [1]. While, the former is aimed at increasing spectrum utilization capacity so as to accommodate more users per spectrum channel [2]. The later, aims to extract a first-hand information on the quality of service in real world scenario. As empirical data are of critical value to the RF and application software engineers.

LTE originally viewed as the potential Fourth Generation (4G) standard by 3GPP. Increasing demand for higher data rates motivated standardization bodies for the development of next generation telecommunication network technologies, which can deliver more data than the previous technologies. LTE network is an all-IP, high throughput and low delay network. To reduce end-to-end delay, the number of vertical network elements is reduced in LTE Release 8 Standard [3]. A low end-to-end delay is one of the most important characteristics of the LTE network, which increases the overall service quality in delivering time-sensitive data traffic. LTE standard promises to deliver $100 \mathrm{Mbps}$ in downlink and $50 \mathrm{Mbps}$ in uplink in $20 \mathrm{MHz}$ bandwidth.

It has been almost three years since the launched of the first LTE network services in Malaysia [4]. However, based on our limited research, there are no research works that analyze the LTE network performance and relate to the theoretical background on key network performance measurements such as RSSI, round-trip time and throughput based on Malaysia environment.

LTE Standard as current run in Malaysia operates on either 1.8 or $2.6 \mathrm{GHz}$ [5]. It is widely known that the gigahertz bands have poor signal propagation characteristics and this is one of the major causes of poor signal quality experienced in the test environment. Obviously, an essential response is herein needed for optimum user satisfaction. A possible solution is to have LTE operate in vacant Television White Space (TVWS). TVWS can result in two processes; (i) analogue to digital TV transmission switch, freeing up channels located in the VHF/UHF spectrum (50-700 MHz) [6], and (ii); currently underutilized TV spectrum. TVWS consist of incumbent users, often known as primary users and the secondary users, referred as opportunistic users to access wireless services and the database operators, to offer the available (or free) UHF/VHF spectrum to the secondary users.

For TVWS to be utilized for wireless communication, spectrum "holes" must be discovered. The discovery of spectrum holes depends on system parameters of incumbent, propagation characteristics and FCC rules [7]. In order to reduce the stringent requirement for the use of TVWS, FCC has approved the services of Database administrators (DBA) so as to ensure interference protection of incumbents. The DBA are further categorized into non-profit seeking operator (e.g. Google [8]) and profit seeking (e.g. Spectrum Bridge [9]). 
There are several on-going research work on the application of TVWS technology as the backbone for broadband communication in several countries such as: Malawi and South-Africa [10]. Furthermore, the National University of Singapore (NUS) is currently undertaken three pilot project on TVWS under the initiative of Singapore White Space Pilot Group (SWSPG). The pilot projects are Smart Metering in National University of Singapore (NUS), Super Wi-Fi Broadband Connectivity in Singapore Island Country Club and broadband connectivity around Changi Airport [11].

The purpose of this paper can be summarized as follow: (i) to provide analysis of a real LTE \& $3 \mathrm{G}$ network using the performance metrics of throughput and RSSI values under mobile vehicular conditions and (ii) determine the TVWS spectrum availability for the potential wireless campus network.

In this paper, we explore fundamental issue pertaining to LTE and $3 \mathrm{G}$ networks data delivery and the subsequent question: what is the actual performance on LTE and $3 G$ networks in sub urban in block terrain? Clearly, to answer this question, performance comparison between the original targets specified in Release 8, introduced in 2009 [12] and the actual live network implementation has to be conducted. Important side benefits of our analysis is that it highlights the need for having $4 \mathrm{G}$ with dual frequency mode of operation.

The rest of paper is organized as follows: Section I introduces the paper and II provide background on the test environment; Section III explains our testing methodology; Section IV presents test results, and Section V conclude this paper.

\section{TVWS TECHNICAL REQUIREMENT}

To freely use TVWS, secondary-to-primary interference must be avoided. This requires the knowledge of TV towers (primary transmitters) and their transmitting parameters. In our case, the TV towers and their location can be readily obtained from spectrum regulatory body of Malaysia, referred as Malaysian Communications and Multimedia Commission (MCMC) [13]. Considering the importance of protecting the primary users from the harmful interference of the secondary users, FCC stipulated additional protection region in conjunction with protection contour as depicted in Fig. 1

FCC stipulates further requirements for a device to use TVWS include: (i) power limit shall not exceed the maximum EIRP of $20 \mathrm{dBm}$ for portable devices and 36 $\mathrm{dBm}$ for fixed device per $6 \mathrm{MHz}$, (ii) transmit antenna height may not be more than 30 meters above the ground. Furthermore, devices making use of TVWS shall protect Personal Land Mobile Radio Services (PLMRS) and Commercial Mobile Radio Services (CMRS) by not operating at a distance less than $134 \mathrm{~km}$ for co-channel and $131 \mathrm{~km}$ for the adjacent channel. Other considerations include; restriction from operating at a distance of $2.4 \mathrm{~km}$ from a registered Radio Astronomy sites. Having identified TVWS technical requirements, we proceed to describe our test environment for both experiments.

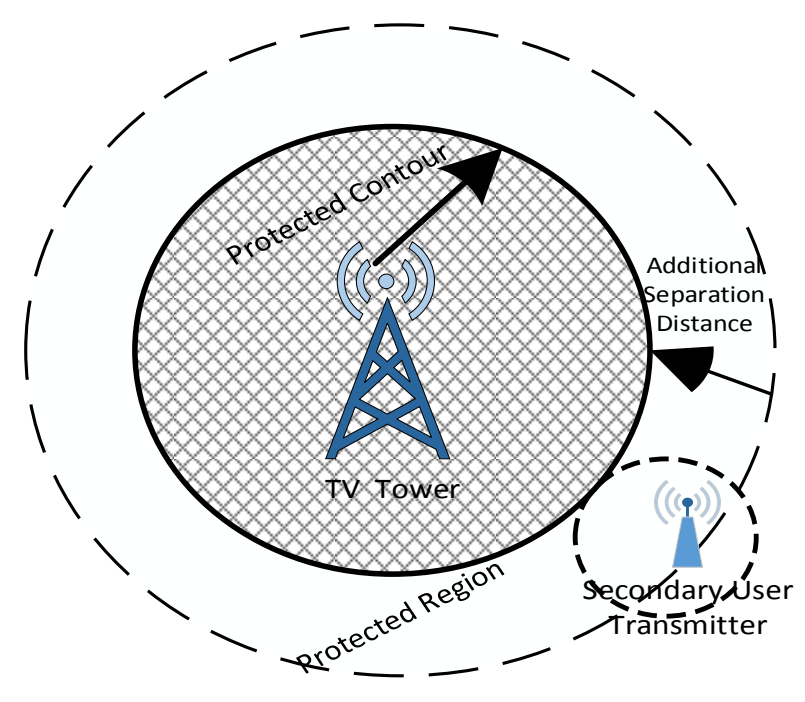

Figure 1: Protected Contour \& Protection Region Defined by FCC [6]

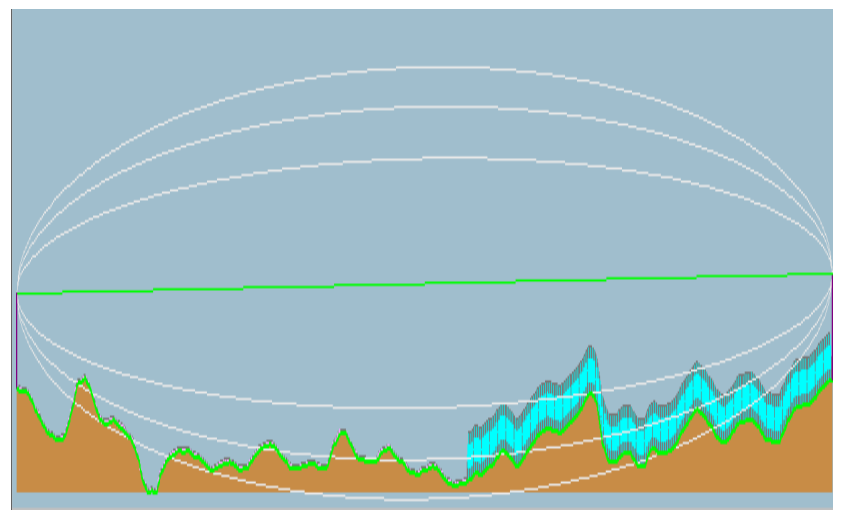

Figure 2: Terrain Profile of Test Environment

\section{The Test Environment And Test Parameters}

The test environment is the campus of Universiti Kebangsaan Malaysia (UKM) campus located in Bangi, Malaysia with coordinate $2.9197^{\circ} \mathrm{N}, 101.7814^{\circ} \mathrm{E}$. The university campus consists of several core containing high rise buildings surrounded by hilly terrains, valley and thick jungle as shown in Fig. 2.

Fig. 2 is simulated using Radio Mobile Online software [14]. This is a radio wave propagation prediction tool that uses digital terrain information and a mathematical model to simulate radio transmissions between two fixed sites. Actual terrain information is crucial for realistic and empirical path loss model [15]. Based on our scenario, the appropriate path loss model that is adequate is the wellknown HATA model to represent sub-urban area around the campus [16].

It is worth mentioning that the drive test was conducted both for the spectrum utilization and expected actual data rate experiments respectively. For the expected actual data rate experiment, the location was concentrated within the 
university campus. The reason being that objective is to characterize the actual data rate experienced within the university campus with $3 \mathrm{G}$ and LTE Release 8 networks. Moreover, for the spectrum utilization, the experiment was conducted within the university campus, freehold property of campus namely UKM KTM train station and a close by bus terminal near the university campus. The importance of taking readings at the bus terminal is to compare the presence of TV signals based on the fact that it is nearby a residential area. We now briefly describe the test parameters of both experiments.

\section{A. LTE Rel. 8 \& HSPA+ Test Parameter Definition}

There are several important measurements in LTE network. These performance metrics give information about the quality of the wireless channel. All of the measurements are conducted over Resource Elements (REs) that contain Reference Symbols (RSs). RSs are transmitted in first and fourth OFDM symbol of each RB, and at each OFDM symbol, the fourth and the sixth REs contains the RS [17]. Measurements are averaged over time and the resource blocks.

Reference Signal Strength Indicator (RSSI) is defined as the total power received in a single Resource Element (RE) in the Resource Block (RB), [17]. RE is defined as the smallest data unit in LTE Standard and represents 15 $\mathrm{kHz}$ in the frequency domain and 1 OFDM symbol in the time domain. One RB contains $12 \mathrm{RE}$, which corresponds to $180 \mathrm{kHz}$ in the frequency domain and $0.5 \mathrm{~ms}$ in the time domain. RSSI measurement includes all of the power coming from all the resources (serving cell, neighbor cells, noise, etc.), and RSSI is the total power coming from all of these resources [18].

A data rate is defined as the quantity of data that can pass from the source to destination in a specific time [19]. In LTE Release 8, the targeted download rate is $100 \mathrm{Mbps}$ for Cat. 3 UE type and $150 \mathrm{Mbps}$ for Cat. 4 UE type, while in HSPA+, the targeted download rates at the UE is 42 Mbps [20]. Round trip time is defined as the time it takes for a single data transaction to occur, meaning the time it takes for the packet of data to travel to and from the destination, back to the source [19]. In LTE Release 8, the round trip time is below $20 \mathrm{~ms}$. In $\mathrm{HSPA}+$, the round trip time is below $25 \mathrm{~ms}$ [19]. Both the data rates \& round trip time for LTE Release 8 \& HSPA+ is summarized in Table I. All the three performance metrics and their target outputs will be compared with the measurement taken by using the TEMS device.

\section{B. TVWS Test Parameter Definition}

Similarly, we conducted spectrum occupancy test for TV channels around and within the campus. In re-using already allocated spectrum, there is a consensus among the major players, which are the industry, licensed spectrum and unlicensed spectrum users that any changes to the FCC spectrum policy must protect the rights of the spectrum owners from harmful interference. Following the industry procedure, test was run in the field using the
TABLE I. OUTPUT SPECIFICATIONS FOR BOTH LTE REL. 8 \& HSPA+

\begin{tabular}{|c|c|c|}
\hline Performance Metrics & LTE Rel. 8 [3] & HSPA+ [9] \\
\hline Data Rates (Mbps) & 100 & 42 \\
\hline Round trip time (ms) & 20 & 25 \\
\hline
\end{tabular}

TABLE II. RF EXPLORER EQUIPMENT SPECIFICATION

\begin{tabular}{|c|c|}
\hline Specification & 3G Combo \\
\hline Spectrum Analyzer Frequency (MHz) & $240-960$ (left) \\
\hline Frequency resolution (KHz) & 1.0 \\
\hline Amplitude resolution (dBm) & 0.5 \\
\hline RF Impedance (ohm) & 50 \\
\hline DSP & Filter/Fast \\
\hline Amplitude stability & $\pm 1 \mathrm{dBm}$ \\
\hline Amplitude accuracy & $16 \mathrm{~V}$ \\
\hline Max RF DC voltage (V) & $32 / 64$ bits \\
\hline Windows PC Client tool & Nagoya Whip 2.4G \\
\hline Type of antenna & $\mathrm{dBm}$ (right) \\
\hline
\end{tabular}

over-the-air (OTA) signals using a low-cost RF equipment. Portable RF Explorer was chosen for this measurement for its user-friendly features, portability, affordable cost and the ability to cover all TV frequencies [21]. The device can scan spectrum frequency from 240 $\mathrm{Hz}$ to $960 \mathrm{MHZ}$ using Nagoya NA-773 antenna. A threshold value of $-94 \mathrm{dBm}$ was selected based on the value suggested by IEEE 802.22 [22] and Carlson Wireless Equipment specifications on Analog TV threshold [23]. Table II depicts the specification of RF Explorer Equipment.

\section{MeTHODOLOGY}

In this section, we describe the procedure for performing the experiments. First, we describe the procedure for performance of the LTE Rel. 8 \& HSPA+ testing experiment and thereafter, the spectrum occupancy test.

\section{A. LTE Rel. 8 \& HSPA+ Test Methodology}

In all test cases, LTE-capable UE is used in a real $3 \mathrm{G}$ and LTE networks. Traffic considered in this study includes FTP Traffic and video traffic, which was created using video sharing websites (YouTube).

For both indoor and outdoor test cases, TEMS Pocket has been used to collect measurements and events of both $3 \mathrm{G}$ and LTE networks. TEMS Pocket is an Android version of the TEMS software [24], which is able to test various types of cellular networks performance, such as, GSM, WCDMA, HSDPA, HSPA+ and LTE. The test scenarios, which can be executed by TEMS Pocket includes detecting RF coverage holes, spots where is the round-trip delay is high, low throughput spots and others.

After the drive test, the collected measurements will be analyzed by using TEMS Investigation, analysis software. The test case was executed in two types of environments; 
the first environment was the outdoor environment which covers 11 zones inside the campus of the Universiti Kebangsaan Malaysia (UKM), as shown in Fig 3. The second test scenario is executed indoor, inside the Faculty of Engineering and Built Environment (FEBE), it includes walk-test for LTE and $3 \mathrm{G}$ coverage inside staff rooms, lecture rooms and laboratories. The whole process of $3 \mathrm{G}$ and LTE Drive tests is described in Fig 4.

Before starting the outdoor and indoor testing scenarios, a simple test is conducted to predict the coverage of both mobile operators (Maxis and Celcom) inside UKM campus, from a website, known as Open Signal [25] to predict both coverage of $3 \mathrm{G}$ and LTE Networks inside the campus, as shown in Figs 5 and 6 respectively. The time to execute the described test scenarios has been chosen to be during the Busy Hour (between 11 am to $1 \mathrm{pm}$ ), where a high number of users are connected and using both $3 \mathrm{G}$ and LTE services.

\section{B. TVWS Spectrum Occupancy Test}

The most critical task for TVWS is the identification of the spectrum holes. Spectrum holes are function of three (3) dimension parameters: time, location and space. Using the three dimensional attributes of TVWS, spectrum occupancy test was conducted. The measurement was performed outdoor at three selected location using portable RF Explorer Spectrum Analyzer and Touchstone Pro Software. In this procedure, 41 channels of Ultra High Frequency (UHF) band was measured at a different time to identify potential TVWS channels available. Besides the availability of TVWS in the campus, our future goal is to deploy TVWS as the future wireless backbone for campus network. This is aimed at provisioning free $\mathrm{WiFi}$ while in transit using TVWS. The campus transportation route is depicted in Fig. 8.

The future campus network is targeting TVWS IEEE 802.22 Standard. The Standard supports the use of TVWS DBA, Consumer Premises Equipment (CPE) and Base Station (BS). The BS will query the DBA for available TVWS channels to use. Then, the BS will communicate with the CPEs on the TVWS channel availability. This will enable students and non-students to enjoy free WiFi while on transit in the campus. The selected locations where inside the Faculty of Engineering and Built Environment (FEBE), KTM-UKM and Hentian Kajang a nearby residential area as demonstrates in Fig. 9.

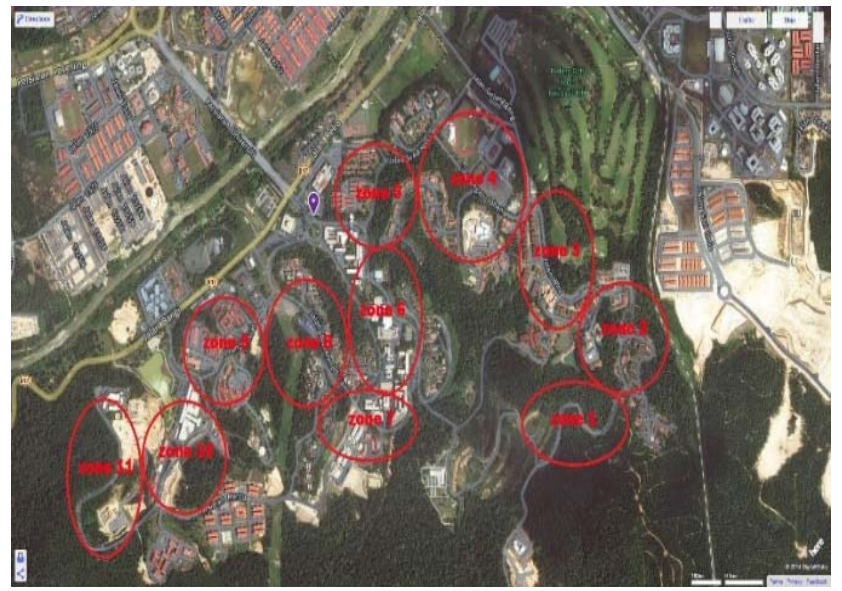

Figure 3: Campus area divided into 11 zone (circled in red)

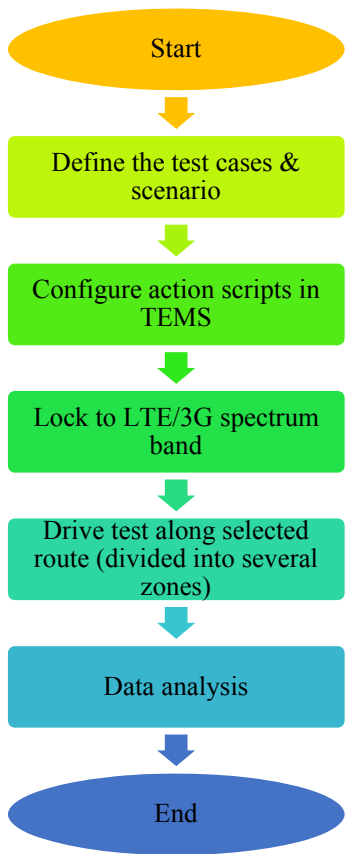

Figure 4: The sequence of drive-test process for both LTE and 3G 


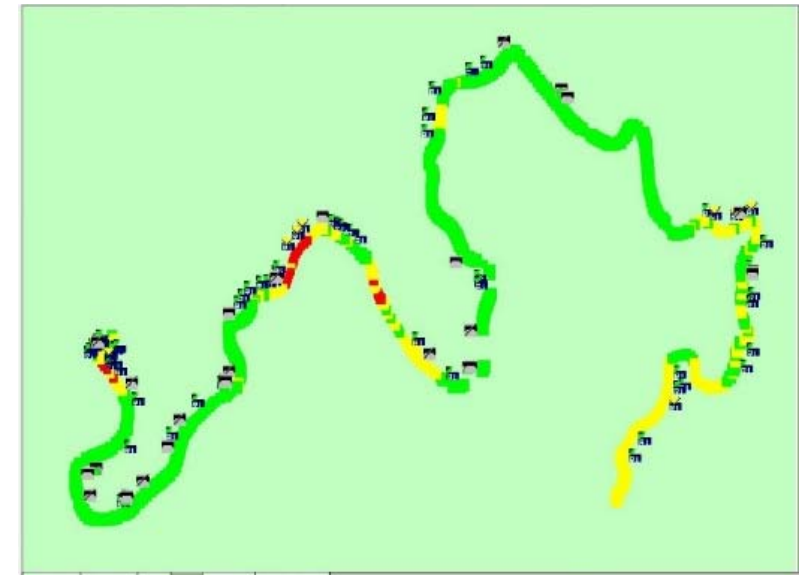

Figure 5: Predefined drive-test route for 11 zones inside UKM campus

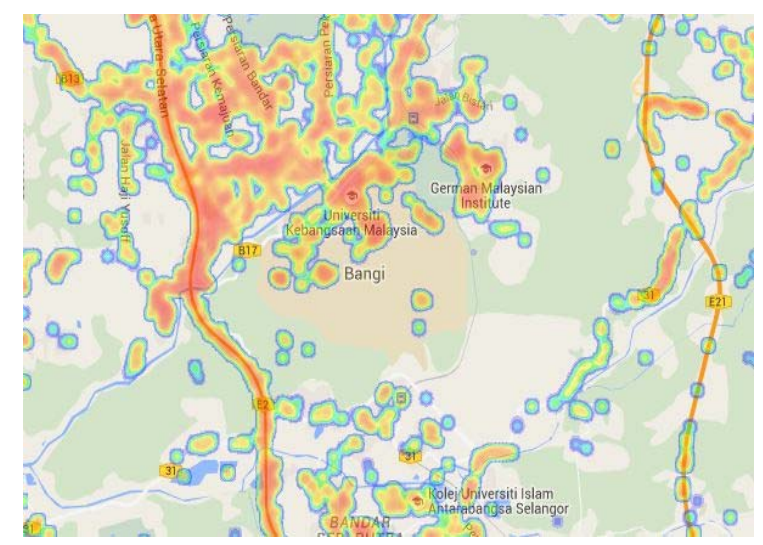

Figure 6: Coverage of Maxis on the campus of UKM [25]

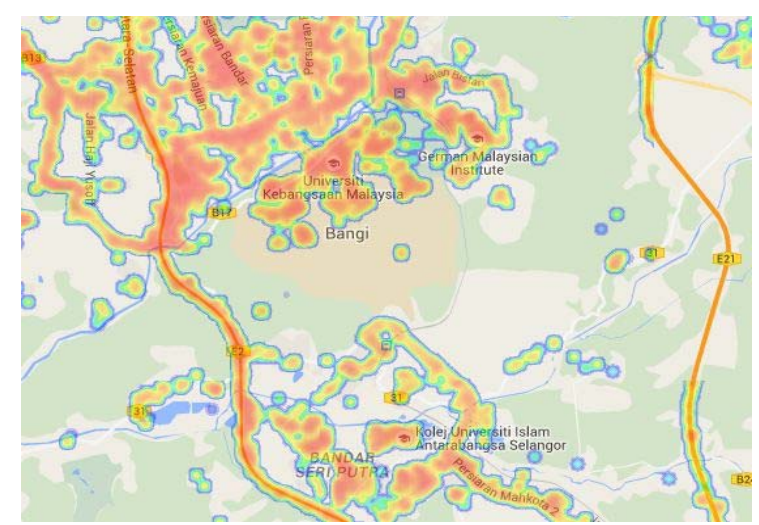

Figure 7: Coverage of Celcom at the UKM campus [25]

\section{RESUlTS AND Discussions}

The following classifications for RSSI measurements which are shown in Table III are adopted for both outdoor and indoor test cases.

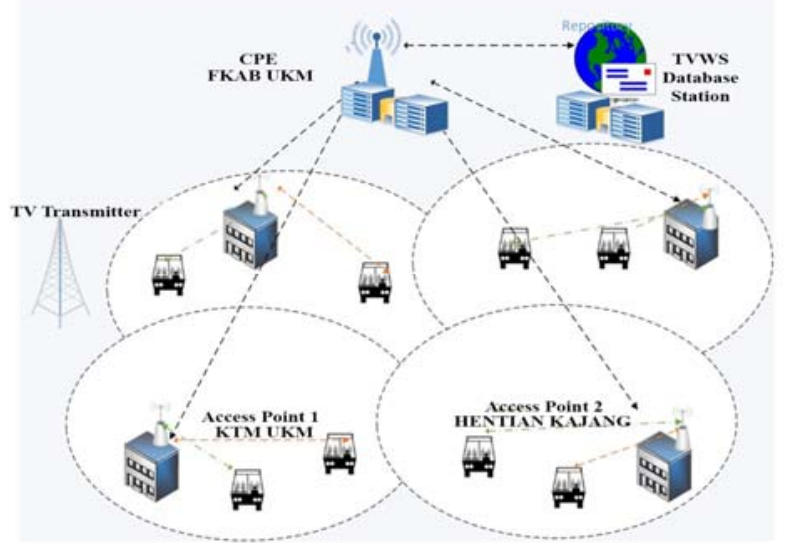

Figure 8: Futuristic IEEE 802.22 Assisted Free Campus WiFi

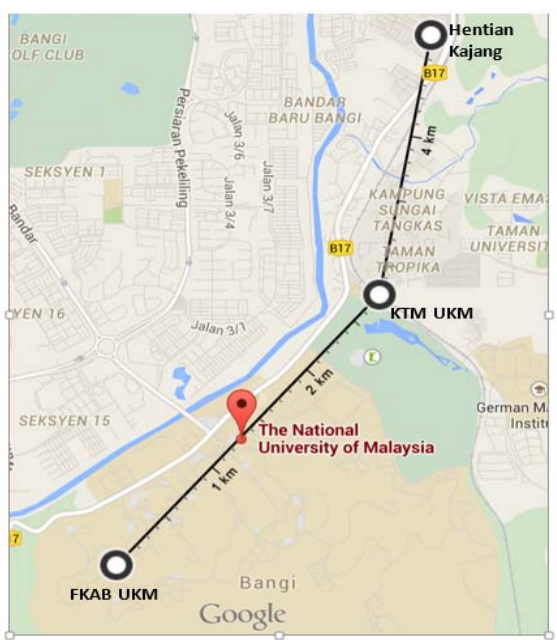

Figure 9: TVWS Measurement location

\section{A. Outdoor Measurements- $3 G$ and LTE}

The first scenario was performed in an outdoor environment. An LTE User Equipment (UE) with TEMS Pocket software was placed in a vehicle and followed a predefined route inside UKM campus as shown in Fig. 5 to record the results. The UE speed was limited between 40 to $50 \mathrm{~km} / \mathrm{h}$ during the tests (campus speed limit), the UE has two antennas and supports two MIMO modes, which is spatial diversity and/or spatial multiplexing.

In spatial diversity mode, the same information is transmitted from both of the antennas of the UE, in spatial multiplexing mode, different information is transmitted to increase the delivered data rate or throughput. In this study, the MIMO configuration is under dual mode. 
TABLE III. CLASSIFICATION OF RSSI MEASUREMENT

\begin{tabular}{|c|l|c|c|}
\hline Classification & \multicolumn{1}{|c|}{ Description } & RSSI Range & Indicator \\
\hline Excellent & $\begin{array}{l}\text { Able to } \\
\text { download/upload to } \\
\text { max data rates } \\
\text { allowed by the } \\
\text { provider \& full } \\
\text { signal reception }\end{array}$ & Above $-70 \mathrm{dBm}$ & Green \\
\hline Good & $\begin{array}{l}\text { No problem } \\
\text { holding a data } \\
\text { connection with } \\
\text { this sort of level }\end{array}$ & $\begin{array}{c}\text { Between }-70 \mathrm{dBm} \\
\text { to }-95 \mathrm{dBm}\end{array}$ & Yellow \\
\hline Poor & $\begin{array}{l}\text { Very likely that } \\
\text { mobile device } \\
\text { suffers low } \\
\text { throughput \& } \\
\text { disconnects due to } \\
\text { cell loading/ } \\
\text { breathing }\end{array}$ & Below $-96 \mathrm{dBm}$ & Red \\
\hline
\end{tabular}

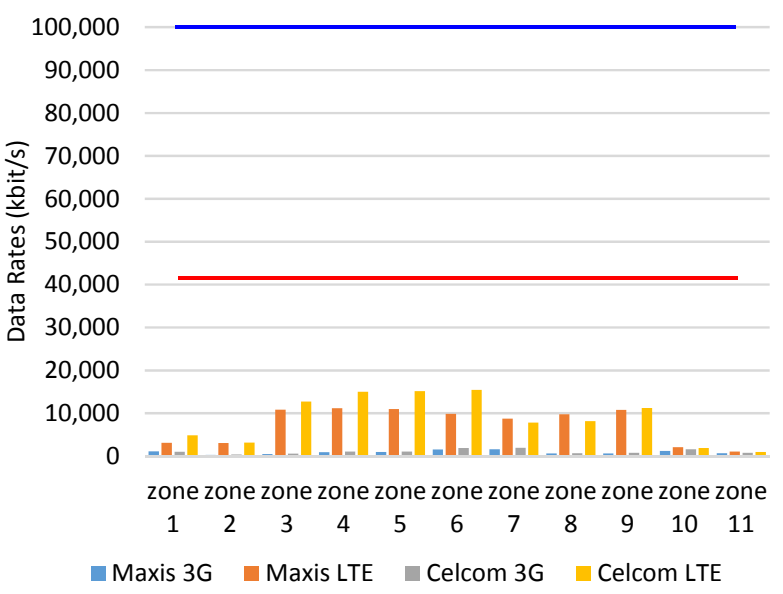

Figure 10: Histogram of user throughput, the blue horizontal line is the targeted LTE user throughout (100 Mbps) and the red horizontal line is the targeted HSPA+ user throughput (42 Mbps)

As shown in Fig. 10, the average throughput that UE receives is varying from one zone to another zone inside the campus. It is clear from Fig. 9 that Zone 1, Zone 2, Zone 10 and Zone 11 has a relatively low user-throughput due to hilly terrain type and the non-Line-of-sight (nLOS) wireless links with the base station in those four zones. However, take note that the highest data rates (Maxis LTE in Zone 6) still far beyond the original LTE target rate, which is $100 \mathrm{Mb} / \mathrm{s}$ for Cat. $3 \mathrm{UE}$ type.

There are several factors that influenced the achievable data rates in live cellular network deployment, compare to the target rates as specified in the documents. These factors can be summarized as follows:

- $\quad$ RF planning \& optimization: eNodeB deployment takes into account plenty of considerations, such as link budget, traffic capacity and on-site limitation, such as location \& height of the sectorized antenna, blockage due to terrain /building, policies from spectrum regulator are some of the factors that limit the performance of an eNodeB. In addition, continuous
RF optimization needs to be scheduled to ensure peak downlink performance

- Application vs. Physical Layer data rates: TEMS only measure the Application Layer data rates, while the expected data rates from LTE Release 8 is based on Physical Layer measurements [26], which include protocol overheads, such as error correction, coding and pilot symbols

- Mobility: in a mobile environment, the vehicle speed is a key factor affecting the data rate of the system. In this case between 40 to $50 \mathrm{~km} / \mathrm{h}$

- Variation in per-user throughput measurement: the number of simultaneous mobile users (network load) influences the peak rates. If the network-level throughput increases, the actual per-user throughput will decrease when the number of simultaneous users increases. Measurement in this study is taken during the peak hours (11 am to $1 \mathrm{pm}$ ), thus the number of simultaneous mobile users is higher than the other period.

- TCP throughput: Various factors affect TCP throughput, such as transfer size, parallel cross traffic (UDP or TCP), the number of established TCP connections, TCP socket buffer size at both ends or possibility of congestion along acknowledgement on the reverse path.

- Link adaptation: Scheduling and adaptive modulation modify the data rates, where users are allocated resources according to the capacity, which has a dependency on current traffic demand and hence affecting throughput.

UE measures RSSI using OFDM symbols that carry reference symbols, and the value are averaged over the time and frequency. RSSI value contains a signal, noise, Rayleigh fading and interference values. From the RSSI measurement, which is shown in Fig. 10, it can be observed that the worst RSSI values for both LTE and 3G were recorded in Zone 1 and Zone 11.
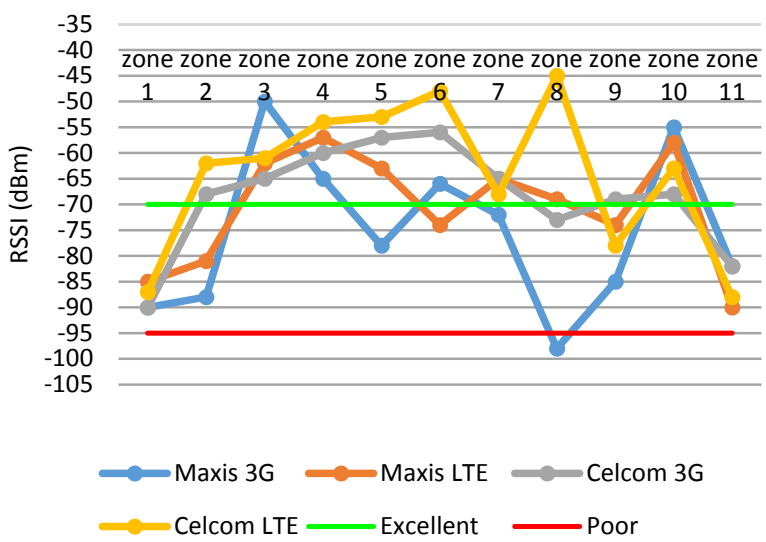

Figure 11: Measured RSSI values for 11 zones 


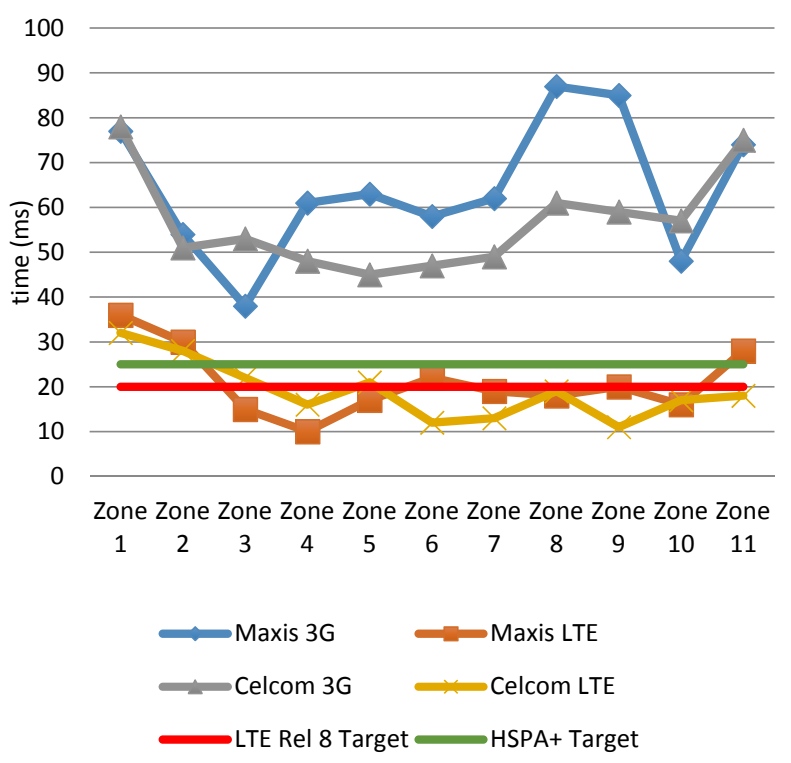

Figure 12. Average Round-Trip Delay (latency) for 3G and LTE [10]

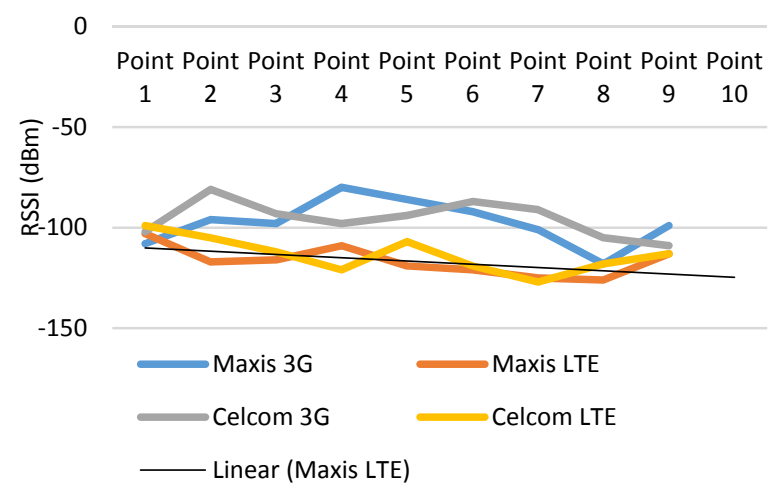

Figure 13. Measured RSSI value for indoor area

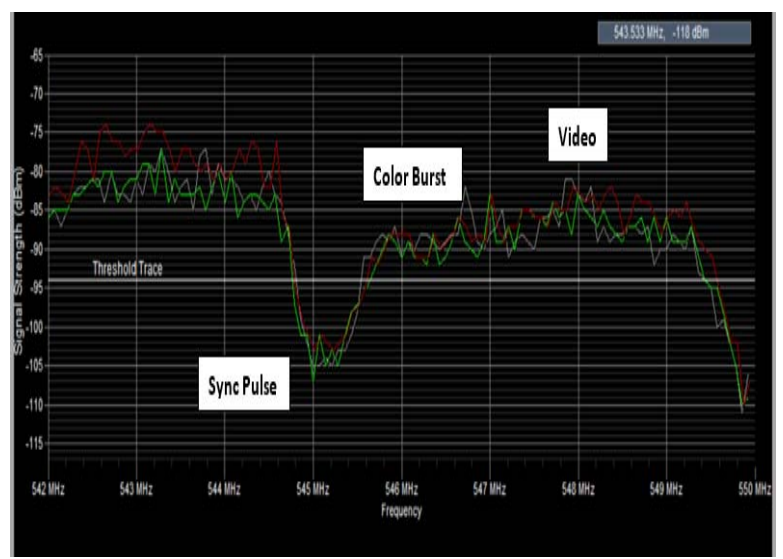

Figure 14: Example of TV RSS measurement at 542MHz
On average, Celcom network shows better coverage for both $3 \mathrm{G}$ and LTE than Maxis network, as shown in Fig 10. This means that Celcom network is very well optimized in terms of the Radio Frequency (RF) planning, network deployment and transmitted power levels and the interference levels for both $3 \mathrm{G}$ and LTE networks. On the other hand, the latency has a major influence on user experience. In particular, conversational services, such as VoIP and video telephony, require low latency.

Other services that benefit from low delay are real-time applications, such as gaming, multimedia streaming and video conferencing. As user requirements for support of new services in a mobile environment is constantly increasing, this is pushing the continuous evolution of mobile technologies. On the other hand, the latency has a major influence on user experience. In particular, conversational services, such as VoIP and video telephony, which require low latency.

The latency in uplink and downlink for both LTE and 3G networks has been tested, Fig. 11. The latency test is called Ping Test in some cases, is simply executed by using the ping command in command interface on Windowsbased platforms. The latency in both uplink and downlink is called round-trip delay. The Transmission Time Interval (TTI) is much shorter in LTE than in $3 \mathrm{G}$ networks, which contributes to improve the latency to be less in LTE in comparison with the $3 \mathrm{G}$ technologies such as HSDPA and HSPA+. The short TTI is considered one factor of so many other factors in LTE, which contributes to lower the latency, such as the compressed UTRAN architecture in LTE, while the $3 \mathrm{G}$ network has larger and centralized RAN architecture. The ping test has shown that both Celcom \& Maxis LTE services almost meet the specified round trip time in LTE Release 8.

\section{B. In-Building Measurements}

The second part of measurements has been carried out for in-building environment, the Faculty of Engineering and the Built Environment (FEBE) at UKM Campus has been chosen to run in-building coverage test for both $3 \mathrm{G}$ and LTE. Ten points are considered for indoor measurement, as shown from the RSSI results in Fig. 9. Celcom showed improved coverage in comparison with LTE when in the building.

Fig. 12 shows that the RSSI values for both mobile service providers, Celcom and Maxis, are much correlated with each other. This is due to more static propagation paths in the indoor environment in comparison with the outdoor environment.

\section{TVWS Spectrum Occupancy Measurements}

Figure 13 shown an example of an occupied TV channel, measured at FKAB. The signal matches a basic composite video signal with signal strength above the threshold margin. Whereas, Figure 14 shown an example of unoccupied TV channel at $710 \mathrm{MHz}$. Noticed that the value is below the threshold of an analogue TV receiver and at the noise floor. 


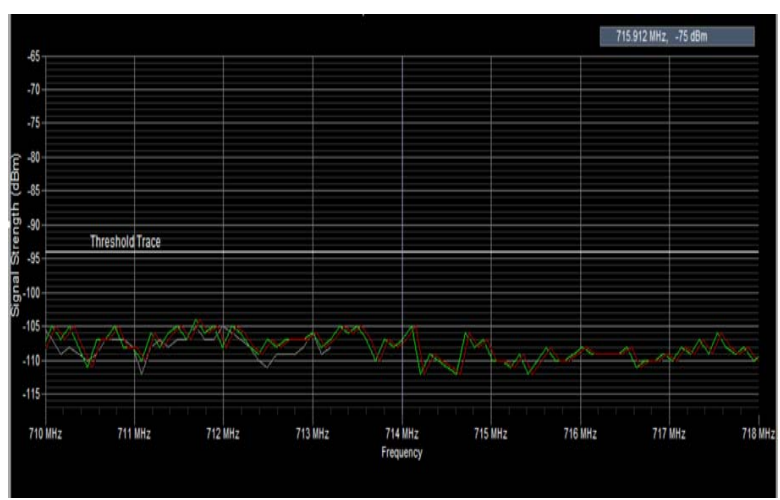

Figure 15: Example of TV RSS Measurement at $710 \mathrm{MHz}$

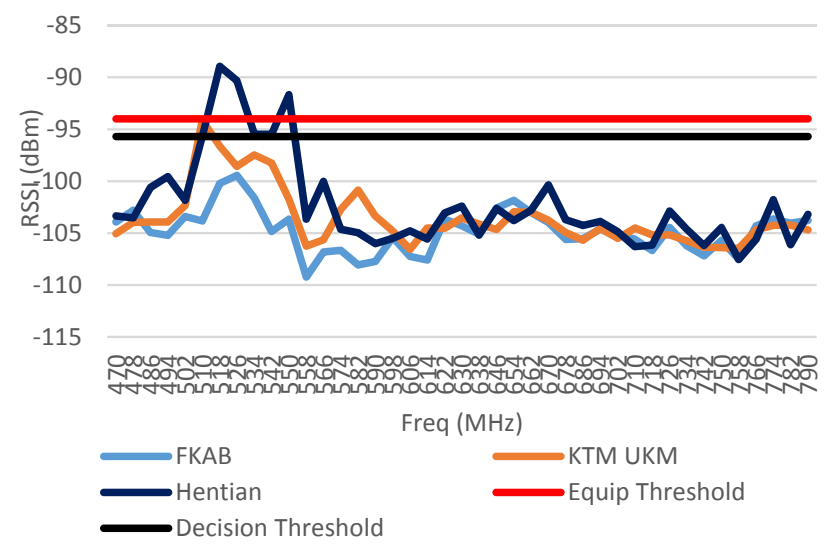

Figure 16: RSSI (dBm) Vs Frequency (MHz)

Figure 15 shows Average RSSI (dBm) vs. frequency of all channels. From this graph, a large number of channels in the frequency range of $646 \mathrm{MHz}$ to $798 \mathrm{MHZ}$ frequency is not being used around the campus area. Note that there are two threshold margins used in this study as explain earlier in Section II.

The other reason for using much lower threshold is to protect TV receivers in worst-case fading scenarios, where a TV signal is received with weak signals. On the other hand, a too conservative settings as suggested by Ofcom will result in a smaller portion of occupancy.

Further analysis finds that the utilization for the threshold of $-94 \mathrm{dBm}$ resulted in $0 \%$ both for FKAB and KTM UKM. $7.31 \%$ for Hentian Kajang. By lowering the threshold value to $-95.68 \mathrm{dBm}$, we found that the utilization is increased at KTM UKM with $2.44 \%$ and Hentian Kajang with $12.20 \%$. This concludes that choosing a slight variation in threshold can result in a number of White Space vacancies found.

\section{CONCLUSIONS}

In this paper, we have conducted two experiments, which are spectrum occupancy test and performance of a real LTE and $3 \mathrm{G}$ networks under mobile conditions, which have been tested for two mobile operators in Malaysia, Celcom and Maxis. The measurement is compared against the original targets of both LTE and HSPA+ networks, and as specified by the standards in LTE Release 8 and 3GPP HSPA+. The UE is installed with ASCOM's TEMS Mobile software and were used in two different test environments; indoor and outdoor. The measurement was performed using the FTP and video traffic test cases. The RSSI values, round trip time and user throughput have been presented and compared. Higher RSSI values lead to higher CQI values, which will result in high throughput values. The test cases were performed in $2 \times 2$ MIMO spatial multiplexing mode, which enforces the transmission of different codewords from each of the antenna.

The coverage of both LTE and $3 \mathrm{G}$ networks appeared to be better on Celcom network, in comparison with Maxis network, which leads to better average throughput delivered by Celcom network. In conclusion, the performance of LTE network changes significantly under mobile conditions, and therefore different approaches are needed to achieve high throughput and optimized coverage in the current and next generation of mobile communications standards.

Conversely, the spectrum occupancy measurement confirms the earlier held notion that most of the licensed UHF channels are unoccupied. Hence, there is a need to exploit this unoccupied TV channels for better wireless service delivery and cheaper (or free) wireless access for the campus students. UHF channels are robust in irregular terrain, where direct line of light is not guaranteed. In addition, the large wavelength size can offer larger coverage radius. This is capable of improving mobile user satisfaction and can be potentially extended to the case of rural wireless broadband.

\section{ACKNOWLEDGMENT}

The authors would like to acknowledge financial support from Malaysia's Ministry of Higher Education, under grant ref no. ERGS/1/2013/ICT03/UKM/02/1.

\section{REFERENCES}

[1] J. Xiao, R. Hu, Y. Qian, L. Gong, and B. Wang, "Expanding LTE network spectrum with cognitive radios: From concept to implementation," Wireless Communications, IEEE, vol. 20, pp. 12$19,2013$.

[2] Federal Communications Commission, "Unlicensed Operations in the TV Broadcast Bands, Second Memorandum Opinion and Order, FCC 10-174," Sept. 23, 2010.

[3] 3GPP TS 36.201, Evolved Universal Terrestrial Radio Access (EUTRA); LTE Physical Layer; General description, Release 8, November, 2007

[4] "Maxis claims first to launch 4G LTE service", Digital News Asia, $2^{\text {nd }} \quad$ Jan, 2013, URL: http://www.digitalnewsasia.com/mobility/maxis-claims-first-tolaunch-4g-lte-service

[5] 3GPP TR 25.913, Version 8.0.0, Release 8, Requirements for Evolved UTRA (E-UTRA) and Evolved UTRAN (E-UTRAN).

[6] R. A. Saeed, TV White Space Spectrum Technologies: Regulations, Standards, and Applications: CRC Press, 2011.

[7] C. Ghosh, S. Roy, and M. B. Rao, "Modeling and validation of channel idleness and spectrum availability for cognitive networks," 
Selected Areas in Communications, IEEE Journal on, vol. 30, pp. 2029-2039, 2012

[8] Google Spectrum Database. [Online]. Available: URL: https://www.google.com/get/spectrumdatabase

[9] Spectrum Bridge Inc. [Online]. Available: URL: http://spectrumbridge.com/ProductsServices/WhiteSpacesSolutions

[10] C. Mikeka, M. Thodi, J. Mlatho, J. Pinifolo, D. Kondwani, L. Momba, et al., "Malawi Television White Spaces (TVWS) Pilot Network Performance Analysis," Journal of Wireless Networking and Communications, vol. 4, pp. 26-32, 2014.

[11] Infocomm Development Authority of Singapore (iDA). Trial of White Space Technology Accessing Vhf And Uhf Bands In Singapore. Available: http://www.ida.gov.sg/doc/Policies and Regulation/Policies and Regulation Level2/WST/WhiteSpaceTP. pdf

[12] 3GPP TR 25.913, Version 8.0.0, Release 8, Requirements for Evolved UTRA (E-UTRA) and Evolved UTRAN (E-UTRAN)

[13] Malaysian Communication and Multimedia Commission (2011)
Press
Release.
[Online].
Available:

http://www.skmm.gov.my/index.php?c=public\&v=art_view\&art_id $=437$

[14] Online. Available: URL: http://www.cplus.org/rmw/rmonline.html.

[15] F. Hessar and S. Roy, "Capacity considerations for secondary networks in tv white space," arXiv preprint arXiv:1304.1785, 2013.

[16] M. Hata, "Empirical formula for propagation loss in land mobile radio services," Vehicular Technology, IEEE Transactions on, vol. 29, pp. 317-325, 1980.

[17] H. Zarrinkoub, Understanding LTE with MATLAB: From Mathematical Modeling to Simulation and Prototyping: John Wiley \& Sons, 2014.

[18] 3GPP TS 25.215, Physical layer Measurements (FDD).

[19] Nokia Siemens Networks, "The impact of latency on application performance", White Paper, 2009

[20] Jodi Zellmer, "HSPA+ and LTE Test Challenges for Multiformat UE Developers", Agilent Technologies, 2012.

[21] M. Zennaro, E. Pietrosemoli, J. Mlatho, M. Thodi, and C. Mikeka, "An assessment study on white spaces in Malawi using affordable tools," in Global Humanitarian Technology Conference (GHTC), 2013 IEEE, 2013, pp. 265-269.

[22] C. Stevenson, G. Chouinard, L. Zhongding, H. Wendong, S. J. Shellhammer, and W. Caldwell, "IEEE 802.22: The first cognitive radio wireless regional area network standard," Communications Magazine, IEEE, vol. 47, pp. 130-138, 2009.

[23] Carlson Inc. URL: http:// carlsonwireless.com.

[24] ASCOM TEMS Pocket, URL: http://www.ascom.com/nt/en/indexnt/tems-products-3/tems-pocket-5.htm

[25] Open Signal, URL: http://opensignal.com

[26] Motorola Inc., "Realistic LTE Performance-From Peak Rate to Subscriber Experience”, White Paper, 2009. 